\title{
La Oferta Educativa Superior del Área de Negocios: Una Perspectiva Internacional Basada en el Ranking Eduniversal
}

\author{
Mónica Hernández-Madrigal ${ }^{*}$, Martha E. Ramos-Córdova y Élfego Ramírez-Flores \\ Facultad de Contaduría y Administración, Universidad Autónoma de San Luis Potosí \\ Madero 849, Colonia Centro. C.P. 78000. San Luis Potosí, S.L.P. México. e-mail: \\ hmadrigalmonica@uaslp.mx,martharamos@uaslp.mx, elfegorf@gmail.com
}

* Autor a quien debe ser dirigida la correspondencia

Recibido Jun. 6, 2013; Aceptado Jul. 18, 2013; Versión final recibida Ago. 28, 2013

\begin{abstract}
Resumen
El objetivo de la presente investigación consiste en analizar la oferta educativa en programas del área económico-administrativa a nivel mundial con base en la proyección y reputación internacional de las principales escuelas de negocios. Se analizan las variables de la oferta educativa de una muestra de 276 escuelas de negocios, públicas y privadas, incluidas en el ranking de EDUNIVERSAL. Los resultados destacan que si bien la proyección internacional no está asociada de forma significativa con nuevas modalidades de educación, con el tipo de régimen jurídico de la universidad ni con el tamaño, existe una asociación significativa con la antigüedad y el nivel económico de los países. Esta investigación pretende contribuir a la literatura educativa ofreciendo reflexiones prácticas que pueden ayudar a las instituciones de educación superior a conocer la importancia de realizar un proceso de comparación con otras universidades a nivel mundial con el fin de mejorar su desempeño.
\end{abstract}

\section{Business Schools Educational Offer: An International Perspective Based on the Eduniversal Ranking}

\begin{abstract}
The objective of this paper is to analyze the economic-management educational programs based on the international reputation of the leading business schools. We analyze the variables of the educational offer in a sample of 276 business schools, both public and private, included in the EDUNIVERSAL ranking. The findings highlight that although international reputation of business schools is not associated with new forms of education, with the type of university nor with the size, there is a significant association with age and economic level of countries. This paper contributes to the educational literature offering practical insights that may help higher education institutions to know the importance of executing a comparative process with other universities in order to improve their performance.
\end{abstract}

Keywords: business schools, international reputation, higher education, educational offer 


\section{INTRODUCCIÓN}

En los últimos años, las nuevas tecnologías, los procesos de transparencia de los organismos, la diversificación de las opciones educativas, el aumento de la competencia, los cambios en el ambiente social, económico y político de los países y la globalización han impactado directamente en el contexto empresarial en el que se desarrollan los profesionales. Así, a la vez que las empresas deben ser capaces de generar riqueza a través de la creación de nuevas fuentes de trabajo y de una actuación eficiente, productiva y socialmente responsable, los profesionales tendrán que ejercer su trabajo apoyándose en una serie de herramientas, conocimientos, competencias y habilidades. En este sentido, la formación de los profesionales ha obligado a las Universidades a adaptar paulatinamente su oferta educativa de acuerdo con las demandas del mercado laboral y con las nuevas oportunidades de negocio.

Por las razones anteriores, se hace necesaria la revisión de la oferta curricular que actualmente ofrecen las universidades. Por un lado, se debe promover una mayor movilidad social de los estudiantes, presentando nuevas opciones de actividad y orientación profesional, y por otro, habrá que llevar a cabo una actualización de los planes y programas de estudio existentes, buscando la creación una nueva cultura educativa que sea capaz de adaptarse a las nuevos requerimientos sociales y a la exigencia de una sociedad en transformación constante.

Uno de los sectores más importantes que contribuye en gran medida al desarrollo de una economía es el educativo (Qureshi et al., 2010). La constante evolución económica, política y social a nivel regional y mundial, a su vez obliga a que la educación cambie rápidamente. Para Lucchesi (2011:34), "un nuevo orden mundial presupone un nuevo orden educacional". La Organización para la Cooperación y el Desarrollo Económico (Organisation for Economic Co-operation and Development, OECD, 2001), define la educación de calidad como aquella que asegura a todos los jóvenes la adquisición de los conocimientos, las capacidades, las destrezas y las actitudes necesarias para prepararles para la vida adulta.

El interés por la calidad en la educación superior comenzó a principios de los años ochenta, incrementándose en los noventa (Mostafa, 2006). La educación superior, como la mayoría de las empresas y organizaciones hoy en día, está cada vez más preocupada por la calidad de sus servicios, debido a un aumento de la competencia y a las exigencias cada vez mayores de los estudiantes y de los grupos de interés en general (Marković, 2006). Por tanto, es importante que las instituciones de educación superior consideren la implementación de prácticas de gestión de calidad como una forma de asegurar su actuación y, al mismo tiempo, conseguir la satisfacción de los estudiantes (De Jager y Gbadamosi, 2010). Así, la administración total de calidad en las instituciones educativas permite pasar de un enfoque de proveer unilateralmente educación superior, a un enfoque de atender una demanda de educación superior (Alharbi, 2009). A este respecto, la mayoría de las universidades a nivel mundial ha intentado incrementar su percepción positiva de calidad a través de la adopción de nuevas modalidades educativas, como una forma de asegurar la satisfacción de los estudiantes (De Jager y Gbadamosi, 2010; Mostafa, 2006).

De acuerdo con Stodnick y Rogers (2008), las universidades están considerando seriamente la evaluación de los servicios de calidad por dos razones esenciales: primera, las recomendaciones verbales juegan un papel importante en su decisión de elegir una universidad y, segunda, los acreditadores de calidad han hecho hincapié en la experiencia del estudiante como uno de sus criterios de evaluación. Para algunos autores, la calidad de la educación se debe medir en términos de reputación, oferta educativa, elección de candidatos, infraestructura física, currículum, ubicación, crecimiento y mejora en los estudiantes, competencias de los docentes, seguimiento de egresados, servicios de librería, flexibilidad de los programas educativos, servicios médicos, nuevas tecnologías, actividades recreativas, alianzas estratégicas, tamaño de los grupos, nivel y dificultad del contenido de las materias y carga de trabajo de los estudiantes (Alharbi, 2009; Barnes, 2007; Cooper, 2007).

La importancia de la calidad a nivel de educación superior se evidencia con el desarrollo de organismos acreditadores nacionales e internacionales que realizan auditorías externas e internas de las instituciones y sus programas (Reid, 2009), por medio de estándares cuantitativos y cualitativos (Jones, 2003). Asimismo, en las últimas dos décadas se ha hecho evidente el surgimiento de rankings comparativos de instituciones educativas que establecen un conjunto de atributos que caracterizan a las universidades, comparándoles entre ellas y confiriendo autenticidad, legitimidad y estatus (Devinney, et al., 2008).

A medida que se buscan políticas educativas efectivas que mejoren las perspectivas económicas y sociales de los individuos, los gobiernos fomentan el desarrollo de comparaciones internacionales que ofrezcan "percepciones valiosas, al permitir que los países vean sus propios sistemas educativos en relación con la calidad, equidad y eficiencia de los servicios educativos logrados en otras partes del mundo" (OECD, 2010:14). Si bien es necesario que los países adopten enfoques colaborativos, cooperativos y consultivos que permitan ofrecer una educación de calidad para los ciudadanos de una nación, es evidente la falta de 
comparaciones educativas entre un país y otro como resultado de las diferencias significativas en la oferta, en los lugares de instrucción y aprendizaje y en el financiamiento (Eskay, 2011). Sin embargo, existe un interés genuino de muchas instituciones de educación por lograr una perspectiva internacional de educación que ofrezca oportunidades para funcionar como una sociedad global (Eskay, 2011). El campo educativo no solo prepara a sus estudiantes para operar en un ambiente global de negocios complejo y dinámico, sino también para operar con las restricciones y los desafíos de dicho ambiente (Bolton y Nie, 2010). Específicamente en el área de negocios, el ambiente en el que se desenvuelven las escuelas es muy competitivo, especialmente ahora que algunas universidades se encuentran compitiendo por menos recursos universitarios (Tullis y Camey, 2007).

\section{METODOLOGÍA}

El objetivo de la presente investigación consiste en analizar la oferta educativa en el área de negocios de distintas universidades con el fin de comparar y contrastar las características de dicha oferta según su nivel de proyección/reputación internacional. La realización de este estudio pretende conocer las tendencias que siguen las escuelas de negocios punteras en cuanto a proyección internacional, específicamente en relación con el tipo de universidad, la antigüedad, el tamaño de la institución y nuevas formas de educación, así como el PIB y la zona geográfica del país donde se ubica.

Así, se establecen las siguientes hipótesis: (a) la proyección internacional de las escuelas de negocios depende del tipo de universidad (pública o privada); (b) existe una asociación significativa entre la proyección internacional y las siguientes características de las escuelas de negocios: antigüedad, PIB, tamaño, tipo de universidad, estatus religioso, modalidad, movilidad, programa de becas, alianzas con otras universidades, exigencia de práctica profesional y flexibilidad de cursos; y (c) hay diferencias significativas entre la proyección internacional de las escuelas de negocios y el área geográfica donde se ubican.

Este estudio se centra en el análisis de la oferta educativa de una muestra extraída a partir de las 1000 mejores universidades de negocios según el ranking EDUNIVERSAL. EDUNIVERSAL (2011) es una empresa francesa de consultoría fundada en 1994 y formada por 12 miembros, los cuales se reúnen para analizar y evaluar el impacto de las tendencias y los cambios globales en materia de educación superior. La empresa ofrece un panorama global sobre las mejores oportunidades educativas en materia de negocios, reconociendo internacionalmente a las mil mejores escuelas en dicho ramo. De acuerdo con EDUNIVERSAL, la reputación internacional es de suma importancia para las escuelas de negocios ya que permite aumentar el valor del alumno y, por lo tanto, mejorar las perspectivas de empleo tanto en su país como en el escenario internacional. EDUNIVERSAL cuenta con un Comité Internacional encargado de la selección oficial de las facultades e integrado por doce miembros: nueve expertos independientes y no remunerados en representación de cada una de las nueve zonas académicas; dos miembros ejecutivos: el Presidente del Consejo de Administración y el coordinador internacional de EDUNIVERSAL, y un miembro consultivo representante del Consejo Académico de las Naciones Unidas (ACUNS).

Por medio del desarrollo de indicadores de calidad, EDUNIVERSAL Ranking localiza y clasifica a las mil mejores escuelas de negocios a nivel internacional, eligiendo determinado número de facultades por país y por continente. El número de escuelas por país es ponderado con base en criterios cuantitativos, tales como el gasto nacional en educación por habitante, el producto interno bruto (PIB), el tamaño de la población, y el número de estudiantes de educación superior, y con base en criterios cualitativos, determinados por el panorama educativo local. A cada una de estas universidades se le asigna, de acuerdo a la puntuación obtenida en la aplicación de la metodología, lo que se denominan "palmas", las cuales pueden ir desde una hasta cinco de acuerdo con la siguiente clasificación (Tabla 1):

Tabla 1: Categorías de escuelas de negocios (EDUNIVERSAL Ranking, 2011)

\begin{tabular}{|c|l|}
\hline № de palmas & \multicolumn{1}{|c|}{ Categoría } \\
\hline 5 & Escuelas de negocios de clase mundial \\
\hline 4 & Mejores escuelas de negocios \\
\hline 3 & Excelentes escuelas de negocios \\
\hline 2 & Buenas escuelas de negocios \\
\hline 1 & Referencias locales \\
\hline
\end{tabular}

La selección de la muestra se basa en las siguientes razones: en primer lugar, dicho ranking ofrece una perspectiva global de la educación superior, lo cual permite comparar las tendencias actuales de la oferta educativa. La segunda razón es porque su enfoque actual se dirige a las escuelas de negocios. En tercer 
lugar, la clasificación de EDUNIVERSAL ranking presenta una segmentación por regiones, lo cual permite llevar a cabo comparaciones entre zonas geográficas. La cuarta razón se basa en el procedimiento de la elección de las universidades con base en criterios de acreditaciones obtenidas y rankings establecidos; en este sentido, las escuelas de negocios contenidas en este ranking han sido evaluadas con base en los mismos criterios, de forma que aquellas empresas que han sido seleccionadas para figurar en el mismo, cumplen con estándares homogéneos de acreditación, lo que proporciona una base más sólida y uniforme en la selección aleatoria de los casos. Finalmente, en la elección de las mil mejores escuelas de negocios participan organizaciones académicas internacionales.

En general, el procedimiento para determinar la selección de las universidades y la asignación de las respectivas palmas responde a los siguientes pasos: primero, el comité científico realiza la selección de escuelas de negocios con base en dos criterios: la universalidad y la proyección internacional de cada centro. El criterio de universalidad hace referencia a la consideración de nueve regiones geográficas a nivel global, que incluyen 154 países. Por su parte, la proyección internacional se refiere a la capacidad de una escuela de negocios para incrementar el prestigio de un estudiante $y$, en consecuencia, mejorar sus posibilidades de empleo tanto en el país donde estudia como a nivel internacional. En segundo término, se asigna la clasificación de las palmas tomando como base dos elementos esenciales: (1) criterios de internacionalización que incluyen las acreditaciones obtenidas por las escuelas bajo análisis (AACSB, EQUIS, AMBA, State Qualification), los principales rankings (Financial Times, Shanghai Jiao Tong, Business Week, Wall Street Journal, America Savings, THES y SMBG), la pertenencia a asociaciones académicas internacionales (EFMD, CLADEA, CEEMAN, EMBA, AAPBS) así como las redes desarrolladas con otras escuelas de negocios; y (2) la votación de los decanos que permite lograr una evaluación entre pares.

Con base en lo anterior, la población objeto de estudio consistió en las mil mejores facultades de negocios de 2008 señaladas en el portal de EDUNIVERSAL. Sin embargo, en dicho portal no se encontró información sobre catorce facultades, por lo que la población final estuvo conformada por 986 escuelas de negocios, repartidas en las distintas regiones del mundo y clasificadas de acuerdo al sitio de Internet EDUNIVERSAL Ranking (Tabla 2).

A partir de dicha población, se calculó una muestra representativa que permitiera determinar la oferta educativa a nivel internacional como sigue:

$n=\frac{Z_{2}^{2}{ }_{2} P Q N}{\varepsilon^{2} N-1+z^{2} P Q}$

Donde $\mathrm{n}=$ tamaño de la muestra, $\mathrm{z}=(\propto / 2)^{\wedge} 2=1.96, \mathrm{P}=$ probabilidad de que el evento ocurra $(0.50), \mathrm{Q}=$ probabilidad de que el evento no ocurra $(0.50), \mathrm{E}=$ error muestral $0.05 \circ 5 \%$ y N = tamaño de la población. La muestra final estuvo constituida por 276 universidades. Posteriormente, con base en la clasificación de EDUNIVERSAL, se llevó a cabo la estratificación de la muestra con el fin de obtener el número de escuelas a analizar por zona geográfica con respecto del total de la población por estrato (Tabla 2).

Tabla 2: Distribución geográfica de las universidades que componen la población y la muestra

\begin{tabular}{|c|l|c|c|c|}
\hline Zona & \multicolumn{1}{|c|}{ Región } & $\begin{array}{c}\text { № de universidades que } \\
\text { integran la población }\end{array}$ & $\begin{array}{c}\text { № de universidades } \\
\text { que integran la muestra }\end{array}$ & $\%$ \\
\hline A & África & 73 & 20 & $7.4 \%$ \\
\hline B & Asia Central & 70 & 20 & $7.1 \%$ \\
\hline C & Europa del Este & 73 & 20 & $7.4 \%$ \\
\hline D & Eurasia y Medio Oriente & 50 & 14 & $5.1 \%$ \\
\hline E & Extremo Oriente de Asia & 140 & 39 & $14.2 \%$ \\
\hline F & Latinoamérica & 100 & 28 & $10.1 \%$ \\
\hline G & Norteamérica & 180 & 51 & $18.3 \%$ \\
\hline H & Oceanía & 50 & 14 & $5.1 \%$ \\
\hline I & Europa Occidental & 250 & 70 & $25.4 \%$ \\
\hline & Total & 986 & 276 & $100.0 \%$ \\
\hline
\end{tabular}


Una vez determinado el número de facultades por zona geográfica, se procedió a elegir, de forma aleatoria, las escuelas de negocios que serían analizadas, utilizando el procedimiento de selección de una muestra aleatoria del programa SPSS (Statistical Package for the Social Sciences - versión 19 para entorno Windows). Cabe mencionar que en tres facultades de negocios no se tuvo acceso a la información de la página web, por lo que las mismas fueron reemplazadas por otras elegidas igualmente de forma aleatoria.

La oferta educativa constituye el conjunto de aspectos y características relacionadas con la enseñanza que se dan a conocer para su consumo, sometida a reglamentos y normas, concretada en materias, programas, horarios y profesores, y expresada en la utilidad que tiene para sus posibles consumidores (Reyes, 2009). En este sentido, se definió una lista que comprende distintas variables de la oferta educativa a analizar de cada una de las facultades de negocios de la muestra. Dichas variables fueron desarrolladas de acuerdo con las últimas tendencias en materia de oferta educativa. Las variables pueden ser clasificadas de la siguiente forma: (1) variables de control como el producto interno bruto del país donde se ubica la escuela de negocios, el tipo de universidad, la antigüedad de la institución, el tamaño de la universidad y el estatus religioso (Zott y Amit, 2008; EDUNIVERSAL Ranking, 2011); (2) variables de desempeño que inciden en el incremento de diferentes formas de educación, en el desarrollo de nuevos proveedores de educación y que tienen un impacto global, tales como la proyección internacional (número de palmas según EDUNIVERSAL Ranking), la movilidad estudiantil (Brooks y Waters, 2010; Collins, 2008), la oferta de becas (McPherson y Schapiro, 1993), las alianzas con otras universidades (Bolton y Nie, 2010), la exigencia de práctica en el mundo laboral (Klimoski, 2007) y la flexibilidad de cursos, basada en la disminución de seriaciones, la incorporación de contenidos y aprendizajes transversales y en la variedad de opciones que se le presentan a los alumnos para que seleccionen diversas materias optativas (Díaz Villa, 2002; Milanovic et al., 2010); y (4) variables tecnológicas como la modalidad de la percepción del aprendizaje (educación a distancia, Collins, 2008; Vlăsceanu y Davies, 2001).

Como medida de desempeño, de resultados y de proyección internacional de las facultades de negocios se ha considerado como variable dependiente el número de palmas asignadas a cada escuela por EDUNIVERSAL. Cabe señalar que para algunas facultades que integran la muestra no se obtuvieron datos de algunas variables. Por tanto, en el análisis de los resultados dichos casos fueron considerados como "casos perdidos" (missing cases). El proceso de evaluación de la información consistió, en un primer paso, en la búsqueda y codificación de la información contenida en cada una de las páginas web de las facultades de negocios seleccionadas de forma aleatoria en la muestra. Dicha evaluación se basó en la lista de variables antes mencionadas. Posteriormente, se desarrolló una base de datos con la información divulgada por cada empresa sobre cada ítem contenido de la lista.

\section{RESULTADOS Y DISCUSIÓN}

Una vez delimitados los objetivos e hipótesis, establecida la metodología de investigación y caracterizada la muestra, se presentan a continuación los principales resultados obtenidos. Por un lado, se llevará a cabo un análisis descriptivo por medio del cálculo de frecuencias, medidas de tendencia central y variaciones y, por otro lado, a fin de verificar las hipótesis de investigación formuladas y establecer relaciones entre los datos, se estudiarán las relaciones causales entre diferentes variables dependientes e independientes, a través de técnicas tales como correlaciones, prueba t de Student y ANOVA (análisis multivariante).

\section{Análisis descriptivo}

Con el objeto de obtener un panorama de las características generales de las escuelas de negocios que forman la muestra, se llevó a cabo un análisis de frecuencias de algunas variables bajo estudio. La Tabla 3 presenta las frecuencias relacionadas con el tipo de universidad, el estatus religioso y la modalidad de enseñanza. Por lo que respecta al tipo de universidad, se encontró que el $46.4 \%$ de las facultades objeto de estudio están sujetas al régimen jurídico público, mientras que el $42.4 \%$ de ellas son de carácter privado. En este sentido, la diferencia proporcional en dicha categoría no es tan alta en comparación con el estatus religioso, encontrando que el $87.3 \%$ de las escuelas de negocios de la muestra son laicas, mientras que el $8.0 \%$ corresponden a asociaciones religiosas.

Por último, en relación a la modalidad de enseñanza que actualmente ofrecen las facultades, se encontró que mientras que el $61.2 \%$ de ellas ofrecen cursos de forma totalmente presencial, solo el $7.6 \%$ se desarrollan de forma virtual. No obstante, un porcentaje considerable (24.6\%) de escuelas de negocios empieza a ofrecer los cursos tanto de forma presencial como virtual. La oferta virtual empieza a tomar relevancia no solo con la finalidad de captar mayores fuentes de financiamiento para las universidades (Koch, 2008; Ressler y Abratt, 2009), sino también como consecuencia del surgimiento de un incremento en la demanda de educación superior, del desarrollo de nuevas tecnologías de información y comunicación, y de los cambios demográficos y globales (Vlăsceanu y Davies, 2001). 
Tabla 3: Frecuencias del tipo de universidad, estatus religioso y modalidad de enseñanza

\begin{tabular}{|l|c|c|l|c|c|l|c|c|}
\hline Tipo de Universidad & № & $\%$ & Estatus religioso & № & $\%$ & Modalidad & No. & $\%$ \\
\hline Pública & 128 & 46.4 & Laica & 241 & 87.3 & Presencial & 169 & 61.2 \\
\hline Privada & 117 & 42.4 & Religiosa & 22 & 8.0 & Virtual & 21 & 7.6 \\
\hline Perdidos & 31 & 11.2 & Perdidos & 13 & 4.7 & Mixta & 68 & 24.6 \\
\hline & & & & & & Perdidos & 18 & 6.5 \\
\hline Total & 276 & 100,0 & Total & 276 & 100,0 & Total & 276 & 100,0 \\
\hline
\end{tabular}

En los últimos años se han desarrollado nuevas formas de educación que ofrecen valor a los estudiantes que buscan tanto movilidad estudiantil, como una dimensión internacional a su experiencia de educación superior (Bolton y Nie, 2010). La transferencia de un programa de una institución a otra o la participación simultánea en dos o más programas o instituciones ha ganado mucha atención en la investigación de la educación superior. En este sentido, la movilidad estudiantil, el otorgamiento de becas, las alianzas con otras universidades, la exigencia de práctica laboral ya sea en el lugar de origen o en otro país, y la flexibilidad de los planes de estudio constituyen parte de la oferta educativa de las escuelas de negocios. En relación al número de palmas obtenidas por las escuelas de negocios de la muestra, se encontró que las de clase mundial (5 palmas) obtienen el porcentaje más alto en relación a la movilidad estudiantil, el otorgamiento de becas, la exigencia de práctica profesional y la flexibilidad de cursos. Asimismo, a medida que disminuye el número de palmas disminuye el número de facultades que implementan estas nuevas formas educativas (Tabla 4).

Tabla 4. Nuevas modalidades de educación superior: frecuencias por palmas

\begin{tabular}{|c|c|c|c|c|c|c|c|c|c|c|}
\hline \multirow{2}{*}{ Palmas } & \multicolumn{2}{|c|}{ Movilidad } & \multicolumn{2}{|c|}{ Becas } & \multicolumn{2}{|c|}{ Alianzas } & \multicolumn{2}{|c|}{$\begin{array}{c}\text { Práctica } \\
\text { professional }\end{array}$} & \multicolumn{2}{|c|}{ Flexibilidad } \\
\hline & No & Sí & No & Sí & No & Sí & No & Sí & No & Sí \\
\hline & & & & & & & & & & \\
\hline 1 & $17.4 \%$ & $82.6 \%$ & $16.7 \%$ & $83.3 \%$ & $33.3 \%$ & $66.7 \%$ & $41.2 \%$ & $58.8 \%$ & $68.2 \%$ & $31.8 \%$ \\
\hline 2 & $9.8 \%$ & $90.2 \%$ & $25.8 \%$ & $74.2 \%$ & $20.5 \%$ & $79.5 \%$ & $29.6 \%$ & $70.4 \%$ & $38.5 \%$ & $61.5 \%$ \\
\hline 3 & $7.4 \%$ & $92.6 \%$ & $23.4 \%$ & $76.6 \%$ & $15.0 \%$ & $85.0 \%$ & $26.6 \%$ & $73.4 \%$ & $35.9 \%$ & $64.1 \%$ \\
\hline 4 & $8.9 \%$ & $91.1 \%$ & $20.4 \%$ & $79.6 \%$ & $12.3 \%$ & $87.7 \%$ & $25.0 \%$ & $75.0 \%$ & $43.4 \%$ & $56.6 \%$ \\
\hline 5 & $0.0 \%$ & $100 \%$ & $3.7 \%$ & $96.3 \%$ & $16.1 \%$ & $83.9 \%$ & $17.4 \%$ & $82.6 \%$ & $15.2 \%$ & $84.8 \%$ \\
\hline Total & $8.1 \%$ & $91.9 \%$ & $20.1 \%$ & $79.9 \%$ & $17.1 \%$ & $82.9 \%$ & $26.8 \%$ & $73.2 \%$ & $38.0 \%$ & $62.0 \%$ \\
\hline
\end{tabular}

Con el fin de obtener un resultado ponderado que abarque las cinco categorías de palmas, se ha obtenido la media y la desviación estándar por zona geográfica. Una media cercana a 5.0 representa una mayor proyección internacional de las facultades. La Tabla 5 muestra que las escuelas de negocios de Oceanía obtuvieron la media más alta (3.93), seguida de Norteamérica (3.69) y Europa Occidental (3.27). En contrapartida, Eurasia y Medio Oriente y África fueron las zonas geográficas que obtuvieron una media de 2,5 y menos (2.50 y 2.25 respectivamente), lo cual indica que las facultades pertenecientes a dichas áreas son consideradas en su mayoría como referencias locales o como buenas escuelas de negocios.

Tabla 5: Estadísticos descriptivos de número de palmas por zona geográfica

\begin{tabular}{|l|c|c|c|c|c|}
\hline \multicolumn{1}{|c|}{ Zona geográfica } & $\mathrm{N}$ & Media & Desv. Est. & Mínimo & Máximo \\
\hline África & 20 & 2.25 & 1.251 & 1 & 5 \\
\hline Asia Central & 20 & 3.05 & 0.887 & 2 & 5 \\
\hline Europa del Este & 20 & 3.05 & 0.826 & 2 & 5 \\
\hline Eurasia y Medio Oriente & 14 & 2.50 & 1.092 & 1 & 4 \\
\hline Extremo Oriente de Asia & 39 & 2.77 & 1.245 & 1 & 5 \\
\hline Latinoamérica & 28 & 2.75 & 1.295 & 1 & 5 \\
\hline Norteamérica & 51 & 3.69 & 0.735 & 3 & 5 \\
\hline Oceanía & 14 & 3.93 & 1.072 & 2 & 5 \\
\hline Europa Occidental & 70 & 3.27 & 0.962 & 1 & 5 \\
\hline Total & 276 & 3.11 & 1.111 & 1 & 5 \\
\hline
\end{tabular}




\section{Análisis multivariante}

Hipótesis: La proyección internacional de las escuelas de negocios depende del tipo de universidad (pública o privada).

De acuerdo con un estudio realizado por la Universidad Nacional Autónoma de México (UNAM) en México, en el área de ciencias las universidades públicas son mejores que las privadas (Martínez Carballo, 2010). Si bien esto sucede en el área de ciencias duras, en el campo económico-administrativo se suele pensar lo contrario. Para obtener evidencia concluyente de la existencia de una relación entre la asignación del número de palmas y el tipo de universidad (pública o privada), se aplicó la prueba t de Student para muestras independientes. Dicha prueba paramétrica fue utilizada debido a que los datos siguen una distribución normal de manera robusta (Figura 1).

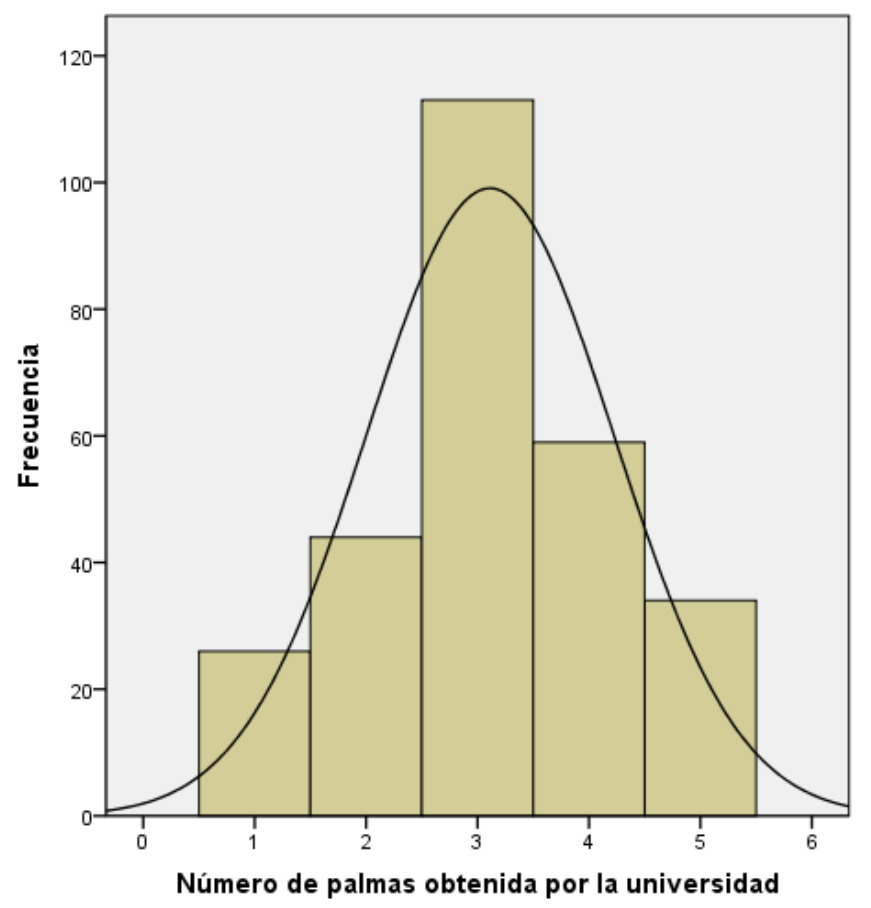

Fig. 1: Distribución normal de los datos según el número de palmas

El valor medio de las facultades de negocios que pertenecen a la universidad pública es de 3.06, mientras que la media de las universidades privadas es de 3.21. Si bien el valor de desempeño de las escuelas de negocios de universidades privadas es mayor que el de las públicas, el nivel de significación estadística es mayor que $0.05(p=0.317)$, lo que significa que la obtención de un mayor nivel de número de palmas no depende del tipo de instituciones. En otras palabras, se puede decir que no existe diferencia significativa a nivel mundial entre la proyección internacional de las universidades públicas y privadas. Por tanto, la proyección internacional no está en función del tipo de universidad, como se suele pensar.

Hipótesis: Existe una asociación significativa entre la proyección internacional y las siguientes características de las escuelas de negocios: antigüedad, PIB, tamaño, estatus religioso, modalidad, movilidad, programa de becas, alianzas con otras universidades, exigencia de práctica profesional y flexibilidad de cursos.

Buscando confirmar asociaciones potenciales entre los diferentes factores que pueden influir en la proyección internacional de las escuelas de negocios, se ha llevado a cabo el análisis de correlación de Spearman para comprobar la existencia de correlación entre la variable dependiente palmas y las variables independientes antigüedad, $P I B$, y tamaño. Como se señaló anteriormente, los datos siguen una distribución normal, por lo que se aplicó la técnica paramétrica de correlación de Pearson. No obstante, si bien los datos siguen una distribución normal, la variable del número de palmas, construida con base en indicadores y consideraciones cualitativas, también da lugar a la utilización de la correlación de Spearman. Cabe señalar que se obtienen resultados similares tanto en la correlación de Pearson como en la de Spearman. Los resultados se muestran en la Tabla 6. 
Tabla 6: Matriz de Correlación de Pearson: proyección internacional / antigüedad, PIB, tamaño. ** La correlación es significativa al nivel 0.01 (bilateral); * La correlación es significativa al nivel 0.05 (bilateral).

\begin{tabular}{|c|c|c|c|c|}
\hline & Palmas & Antigüedad & PIB & Tamaño \\
\hline Palmas & 1.000 & $0.217^{* *}$ & $0.368^{* *}$ & 0.109 \\
& & $(0.000)$ & $(0.000)$ & $(0.136)$ \\
\hline \multirow{2}{*}{ Antigüedad } & & 1.000 & $0.349^{* *}$ & $0.308^{\star *}$ \\
& & & $0.000)$ & $(0.000)$ \\
\hline \multirow{2}{*}{ PIB } & & & 1.000 & $0.156^{*}$ \\
& & & & $(0.033)$ \\
\hline \multirow{2}{*}{ Tamaño } & & & & 1.000 \\
\hline
\end{tabular}

En términos generales, los resultados indican que hay una relación positiva entre el nivel de proyección internacional y las tres variables bajo estudio. Sin embargo, las variables antigüedad y PIB muestran el índice de correlación más alto respecto al nivel de proyección internacional medido en palmas $(0.217$ y 0.368 respectivamente) y un valor estadísticamente significativo $(p<0,05)$. En contrapartida, la variable tamaño muestra un valor significativo bajo $(p>0,05)$, así como un bajo índice de correlación. Cabe destacar que la variable PIB muestra la correlación más alta (0.368), lo cual a su vez indica que mientras más potencial económico tenga el país, las escuelas de negocios desarrollarán una mayor reputación internacional. En línea con lo anterior, Little (2003) destaca que en los estudios internacionales comparativos sobre educación, se debe tomar en cuenta que las mayores ideas y políticas sobre educación se originaron en el Norte, es decir en los países desarrollados.

Adicionalmente, con el fin de obtener evidencia sobre la existencia de una relación entre la proyección internacional y las nuevas modalidades de la educación superior, tales como la movilidad, el programa generalizado de becas, las alianzas con otras universidades, la exigencia de práctica profesional y la flexibilidad de los cursos, además del estatus religioso y la modalidad (considerada solo como presencial o virtual), se llevó a cabo el análisis de comparación de medias de muestras independientes por medio de la prueba t de Student para distribuciones normales. Dicha prueba fue seleccionada debido a que las variables independientes (modalidades, estatus religioso y modalidad) son dicotómicas (sí o no) mientras que la variable dependiente (proyección internacional) es de carácter ordinal. Así, los resultados indican que no existen diferencias significativas a nivel internacional entre la proyección internacional y los nuevos métodos de enseñanza, el estatus religioso y la modalidad, ya que en todas las pruebas el nivel de significación estadística fue mayor a $0.05(0.752,0.865,0.184,0.850,0.903,0.310$ y 0.325 respectivamente).

Hipótesis: Hay diferencias significativas entre la proyección internacional de las escuelas de negocios y el área geográfica donde se ubican.

Para determinar si existen diferencias significativas entre la media de la proyección internacional de la escuela de negocios en relación al área geográfica donde se ubica, se llevó a cabo un análisis de varianzas (ANOVA) con un nivel de confianza del 95\% para la distribución normal. Se seleccionó dicho análisis debido al carácter ordinal de la variable dependiente (palmas) y al carácter multitómico de la variable independiente (zona geográfica). Como puede observarse en la Tabla 5, las zonas geográficas de Oceanía y de Norteamérica muestran las medias de proyección internacional más altas (3.93 y 3.69 respectivamente) en comparación con las zonas restantes. En este sentido, la prueba de homogeneidad de las medias (Tabla 7) indica un valor significativo menor que $0.05(0.000)$, por lo que las medias entre las zonas geográficas son significativamente diferentes.

Estos resultados son consistentes con los obtenidos en la hipótesis anterior, ya que las zonas geográficas que muestran un nivel más alto de proyección internacional son aquellas cuyo PIB es mayor (Oceanía, Norteamérica y Europa Occidental), en contrapartida con las escuelas de negocios que muestran una proyección internacional no tan alta, ubicadas en zonas cuyo PIB es bajo (África y Eurasia y Medio Oriente). Por tanto, sería interesante investigar las asociaciones causales de cada área geográfica. En síntesis, son significativas las diferencias de proyección internacional entre las distintas regiones del mundo.

Con el fin de conocer dónde se encuentran las diferencias significativas entre las zonas geográficas, se realizó una comparación por pares aplicando la prueba discrecional de Tukey. Dicha prueba permite medir la diferencia de los valores de las medias de dos grupos. Así, se encontró que las áreas que difieren de forma significativa son, por un lado, las zonas de África, Eurasia y Medio Oriente, Extremo Oriente de Asia y Latinoamérica con respecto a las zonas geográficas de Norteamérica y Oceanía. 
Tabla 7: Anova de un factor: proyección internacional / zona geográfica

\begin{tabular}{|l|c|c|c|c|c|}
\hline & Suma de cuadrados & Gl & Media cuadrática & F & Sig. \\
\hline Inter-grupos & 56.443 & 8 & 7.055 & 6.655 & 0.000 \\
\hline Intra-grupos & 283.075 & 267 & 1.060 & & \\
\hline Total & 339.518 & 275 & & & \\
\hline
\end{tabular}

\section{CONCLUSIONES}

1.- Se destaca la importancia de la implementación de nuevas formas de educación en la oferta educativa, tales como la movilidad estudiantil, el otorgamiento de becas, las alianzas con otras universidades, la exigencia de práctica laboral ya sea en el lugar de origen o en otro país, y la flexibilidad de los planes de estudio. En cuanto al concepto de proyección internacional, las escuelas de negocios que ocupan los primeros puestos del ranking han adaptado su oferta educativa a las nuevas exigencias y demandas de los cambios de la sociedad.

2.- Los resultados indican que la proyección internacional de las escuelas de negocios no está asociada con el tipo de régimen jurídico de la universidad. Este hecho enfatiza la pertinencia de la inclusión tanto de universidades públicas como privadas en el estudio, al tener las mismas oportunidades de sobresalir como institución. Las universidades públicas compiten en calidad con cualquier tipo de institución privada.

3.- Si bien el tamaño de las escuelas de negocios no muestra una asociación significativa con el nivel de proyección internacional de las facultades, la proyección internacional de las escuelas de negocios sí está significativamente relacionada con la antigüedad y con el PIB de los países donde se ubican dichas escuelas. Con respecto a este último punto, se constata la relevancia de los países desarrollados en el proceso de implementación de nuevas estrategias educativas, así como el liderazgo en procesos innovadores relacionados con la oferta educativa.

Este artículo contribuye a la literatura educativa ofreciendo reflexiones prácticas sobre las tendencias a nivel global de la oferta educativa de las escuelas de negocios que se resumen, de forma general, en dos opciones: apostar por un compromiso con la innovación o apostar por resultados probados siguiendo la línea de las universidades punteras.

\section{REFERENCIAS}

Alharbi, M. Evaluation of quality: Student Perceptions, The International Journal of Learning: 16(9), 281-296 (2009).

Barnes, B.R. Analysing service quality: the case of post-graduate Chinese students, Total Quality Management: 18(3), 313-331 (2007).

Bolton, D. y R. Nie. Creating value in transnational higher education: the role of stakeholder management, Academy of Management Learning \& Education: 9(4), 701-714 (2010).

Brooks, R. y J. Waters. Social networks and educational mobility: the experiences of UK students, Globalisation, Societies and Education: 8(1), 143-157 (2010).

Collins, F.L. Bridges to learning: international student mobilities, education agencies and inter-personal networks, Global Networks: 8(4), 398-417 (2008).

Cooper, P. Knowing your 'Lemons': Quality uncertainty in UK higher education, Quality in Higher Education: 13(1), 19-29 (2007).

De Jager, J. y G. Gbadamosi. Specific remedy for specific problem: measuring service quality in South African higher education, Higher Education: 60(3), 251-267 (2010).

Devinney, T., G.R. Dowling y N. Perm-Ajchariyawong. The Financial Times business schools ranking: What quality is this signal of quality? European Management Review: 5(4), 195-208 (2008).

Díaz Villa, M. Flexibilidad y Educación Superior en Colombia, (2002), http://ambiental.uas/p.mx/desc/DiazVilla-FlexibilidadEducacionV2.zip Acceso: 07 de Agosto (2013).

EDUNIVERSAL. The University and Business School Ranking Worldwide (2011). http://www.eduniversalranking.com/ Acceso: 2 de Julio (2011). 
Eskay, M. Harmonizing educational differences from an international perspective, Cypriot Journal of Educational Sciences: 6(1), 30-36 (2011).

Jones, S. Measuring the quality of higher education: linking teaching quality measures at the delivery level to administrative measures at the university level, Quality in Higher Education: 9(3), 223-229 (2003).

Klimoski, R. J. From the editor: Becoming a prophet in our own land, Academy of Management Learning \& Education: 6(4), 433-438 (2007).

Koch, J.V. The relative decline of a Musgrave 'Merit Good:' the case of public support of flagship public universities, Journal of Economics and Finance: 32(4), 368-379 (2008).

Little, A. W. Motivating learning and the development of human capital, Compare: 33(4), 437-452 (2003).

Lucchesi, M.A.S. La Universidad Internacional en América Latina: un Nuevo Paradigma para el Siglo XXI, Formación Universitaria: 4(1), 25-36 (2011).

Marković, S. Expected service quality measurement in tourism higher education, Pregledni Znanstveni Clanki / Review Papers: 1-2, 86-95 (2006).

Martínez Carballo, N. Pide SEP 'creatividad e imaginación’ ante crisis, (2008).

http://www.eluniversal.com.mx/sociedad/1177.html Acceso: 14 de Septiembre de 2011

McPherson, M. S. y M.O. Schapiro. The effect of government financing on the behavior of colleges and universities. En M. S. McPherson, M. O. Schapiro, y G. C. Winston, Paying the piper: Productivity, incentives, and financing in U.S. higher education (235-250). MI: University of Michigan Press (1993).

Milanovic, I., T.A. Eppes, J. Girouard y L. Townsend. Retention-oriented curricular design, Journal of College Teaching \& Learning: 7(11), 41-46 (2010).

Mostafa, M.M. A comparison of SERVQUAL and I-P analysis: measuring and improving service quality in Egyptian private universities, Journal of Marketing of Higher Education: 16(2), 83-104 (2006).

Organisation for Economic Co-operation and Development (OECD). Schooling for Tomorrow: Trends and scenarios. OECD Publications, Paris, Francia (2001).

Organisation for Economic Co-operation and Development (OECD). Education at a Glance 2010. OECD Indicators. OECD Publications, Paris, Francia (2010).

Qureshi, T.M., M.Z. Shaukat y S.T. Hijazi. Service quality SERVQUAL model in higher educational institutions, what factors are to be considered? Interdisciplinary Journal of Contemporary Research in Business: 2(5), 281-290 (2010).

Reid, I.C. The contradictory managerialism of university quality assurance, Journal of Education Policy: 24(5), 575-593 (2009).

Ressler, J. y R. Abratt. Assessing the impact of university reputation on stakeholder intentions, Journal of General Management: 35(1), 35-45 (2009).

Reyes, R. (Dir.). Diccionario Crítico de Ciencias Sociales. Universidad Complutense de Madrid, Madrid, España (2009).

Stodnick, M. y P. Rogers. Using SERVQUAL to measure the quality of the classroom experience, Decision Sciences Journal of Innovative Education: 6(1), 115-133 (2008).

Tullis, K.J. y J.P. Camey. Strategic implications of specialized business school accreditation: end of the line for some business education programs? Journal of Education for Business: 83(1), 45-51 (2007).

Vlăsceanu, L. y J.L. Davies. Virtual and Classical Universities between Complementarity and Contrast, Higher Education in Europe: 26(4), 475-485 (2001).

Zott, C. y R. Amit. The fit between product market strategy and business model: implications for firm performance, Strategic Management Journal: 29(1), 1-26 (2008). 